Ul trast ruct ural anal ysi s of I i pi d i ncorpor at i on i $n$ the entbryoni $c$ si I kworm Bombyx mori.

\begin{tabular}{|l|l|}
\hline 著者 & $\begin{array}{l}\text { 山濱 由美, Mur anaka Yoshi nor i, Kumaki r i Yoko, } \\
\text { Tanøt su Sat oshi, Har i yama Takahi ko }\end{array}$ \\
\hline $\begin{array}{l}\text { j our nal or } \\
\text { publ i cat i on ti tl e }\end{array}$ & Zool ogi cal Sci ence \\
\hline vol une & 26 \\
\hline nunber & 5 \\
\hline page range & $321-324$ \\
\hline year & $2009-03$ \\
\hline URL & ht t p: //hdl .handl e. net /10271/2077 \\
\hline
\end{tabular}




\title{
Ultrastructural Analysis of Lipid Incorporation in the Embryonic Silkworm, Bombyx mori
}

\author{
Yumi Yamahama $^{1 *}$, Yoshinori Muranaka², Yoko Kumakiri², \\ Satoshi Tamotsu ${ }^{3}$ and Takahiko Hariyama ${ }^{1}$ \\ ${ }^{1}$ Department of Biology, Faculty of Medicine, Hamamatsu University School of Medicine, \\ 1-20-1 Handayama, Higashi-ku, Hamamatsu, Shizuoka 431-3192, Japan \\ ${ }^{2}$ Laboratory for Electron Microscopy, Research Equipment Center, Hamamatsu \\ University School of Medicine, 1-20-1 Handayama, Higashi-ku, \\ Hamamatsu, Shizuoka 431-3192, Japan \\ ${ }^{3}$ Graduate School of Humanities and Sciences, Nara Women's University, \\ Nishiuoyacho, Nara, Nara 630-8903, Japan
}

\begin{abstract}
Insect eggs store many lipid droplets as an energy source for embryonic development. We previously reported that lipid droplets are incorporated into embryos in three steps in the silkworm, Bombyx mori. The midgut plays important roles in lipid incorporation during the second and third steps, whereas the manner of lipid incorporation during the first step is still unknown. In this study, we focused on how lipids were incorporated into the embryo in the first step, compared with the mechanisms used in the second step, by means of transmission electron microscopy using the high-pressure freezing and freeze substitution method. At the beginning of the first step (blastoderm formation stage), some lipid droplets were observed in each cell of the embryonic tissues. Lipid droplets were seen to be derived from the oocyte peripheral cytoplasm by superficial cleavage. At the end of the first step (late appendage formation stage), some lipid droplets were attached to the elongated rough endoplasmic reticulum ( $r E R)$. It seemed that formation of the lipid droplets occurred in embryonic cells at the end of the first step, because the rER is the site of biogenesis of lipid droplets. The incorporation of lipid droplets in the first step may be subdivided into two stages: the blastoderm formation stage and the subsequent stage before blastokinesis.
\end{abstract}

Key words: Bombyx mori, lipid droplet, embryonic development, endoplasmic reticulum, electron microscopy, high-pressure freezing, freeze substitution

\section{INTRODUCTION}

In insects, a large amount of nutrients, such as yolk granules, glycogen granules, and lipid droplets, are stored in the egg. Yolk granules are hydrolyzed and then used as a protein source during embryonic development. Lipid droplets and glycogen granules are used as energy sources during embryonic development; in particular, lipid droplets are digested mainly in the last stage of embryonic development (Beenakkers et al., 1981). The eggs of the silkworm, Bombyx mori, which contain many lipid droplets and glycogen granules, are suitable for studying the energy supply during embryonic development. We recently reported that lipid droplets are incorporated into embryonic tissues in three steps during embryonic development in B. mori (Yamahama et al., 2008). Small amounts of lipid droplets are incorporated into embryonic tissues before blastokinesis (first step), half the lipid droplets in the extraembryonic yolk are incorporated into the embryo by dorsal closure during blasto-

\footnotetext{
* Corresponding author. Phone: +81-53-435-2351; Fax : +81-53-435-2351; E-mail: hamahama@ hama-med.ac.jp
}

kinesis (second step), and the remaining half are eaten by the developing caterpillar before head pigmentation (third step). We supposed that lipid is incorporated in the first step in a different manner than in the other steps, in which lipid incorporation is mediated by the midgut. To determine the mechanism of lipid incorporation during the first step, we made ultrastructural observations.

Miya et al. (1972) and Miya (1976, 2003) previously examined $B$. mori eggs by transmission electron microscopy (TEM), but there have been few recent reports of ultrastructural studies. The mature eggs of $B$. mori are especially difficult to prepare for TEM. Structural changes readily occur, because the cytoplasmic components are easily disordered when the lipid droplets that occupy the large part of the cytoplasm are removed during conventional dehydration processes. We recently applied high-pressure freezing combined with freeze substitution to the preparation of mature eggs of $B$. mori for TEM observation, achieving results suitable for the observation of fine structures such as lipid droplets and glycogen granules (Yamahama, 2008). We also used these methods to determine how lipid droplets are incorporated into embryonic tissues in the first step as compared with the second step. 


\section{MATERIALS AND METHODS}

Animals

Non-diapause eggs of a bivoltine race (Daizo, NIAS Stock No.338) of the silkworm, B. mori, were prepared using a previously described method (Yamahama et al., 2008). All of the eggs were collected within $1 \mathrm{hr}$ after oviposition, and were kept in dark conditions at $25^{\circ} \mathrm{C}$ until hatching. The larvae hatched out on the 9th day after oviposition under these experimental conditions. The developmental stages of the embryos were defined morphologically by referring to Ohtsuki (1979), Yamashita and Yaginuma (1991), and Singh et al. (2002).

We divided the embryos into four groups, as shown in Fig. 1. The chorion was removed in Graces's insect culture medium (Daigo, Nippon Seiyaku Co., Japan) by using a fine needle (27G $\times 3 / 4$; Terumo Co., Japan) under a dissection microscope, just before applying high-pressure freezing.

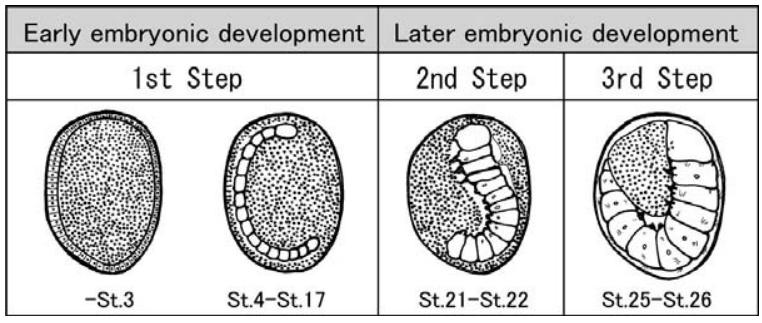

Fig. 1. Schematic drawings correlating the three steps of lipid incorporation with the stages of embryonic development.

\section{Preparation of specimens for electron microscopy by using high-pressure freezing combined with freeze substitution}

Specimens were frozen in a high-pressure freezer (EM-PACT; Leica, Germany) using a processed flat carrier, as previously reported (Yamahama, 2008). Briefly, the hollow of a commercially available flat carrier (Leica, Germany) was additionally processed with a milling machine (Mr. Mister FV-10M; Toyo Associates, Japan) to a size of $1.2 \mathrm{~mm}$ in diameter and $375 \mu \mathrm{m}$ in depth to fit an egg. A dechorionated egg was then gently placed in the hollow of the carrier and immediately frozen in the high-pressure freezer. Freeze substitution was performed by using a freeze substitution freezer (EM-AFS2; Leica, Germany). Frozen-eggs were transferred into substitution solution (1\% osmium tetroxide in $100 \%$ acetone) below $-90^{\circ} \mathrm{C}$, and the substitutions were then performed automatically. After being rinsed with acetone at room temperature, specimens were substituted with Quetol 812 (Nissin EM, Japan) resin. Ultrathin sections were made with an ultramicrotome (UCT; Leica, Germany), picked up on a copper grid covered with collodion film, stained with $2 \%$ uranyl acetate for 5 min followed by $3 \mathrm{~min}$ in lead stain solution (Sigma-Aldrich, USA), and observed by transmission electron microscopy (JEM-1220; JEOL, Japan); digital images were captured with an attached cooled CCD camera (Gatan, USA).

\section{RESULTS}

Transmission electron micrographs of embryonic cells of B. mori during the first step are shown in Figs. 2 and 3. At the beginning of the first step (Fig. 2), in the blastoderm formation stage (stage 3), superficial cleavage had occurred; a monolayer of blastodermal tissues covered the egg surface (see the schematic drawing in Fig. 1). There were some lipid droplets and many glycogen granules in the cytoplasms of blastodermal cells (Fig. 2A), and the rough endoplasmic reticulum $(\mathrm{rER})$ was not well developed (Fig. 2B). At the end of the first

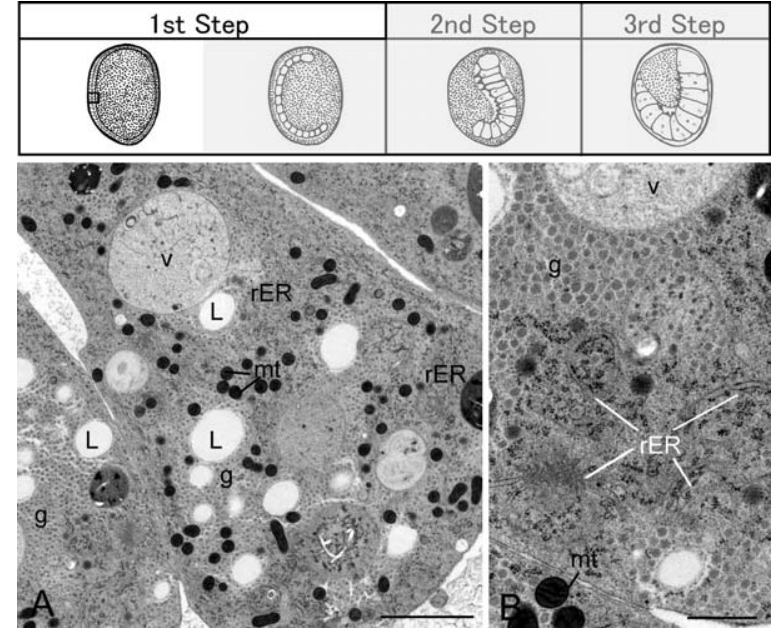

Fig. 2. TEM images of embryonic tissues at the beginning of the first step of lipid incorporation, the blastoderm formation stage (stage 3). (A) Embryonic tissues facing the extraembryonic yolk. (B) High-magnification image of an embryonic cell. The non-shaded panel in the diagram at the top indicates the step of lipid incorporation into the embryo, and the black bold square on the drawing of the embryo indicates the area observed by TEM. L, lipid droplet; $g$, glycogen granule; mt, mitochondria; rER, rough ER; $v$, vacuole. Scale bars, $2 \mu \mathrm{m}(\mathrm{A}), 0.6 \mu \mathrm{m}(\mathrm{B})$.

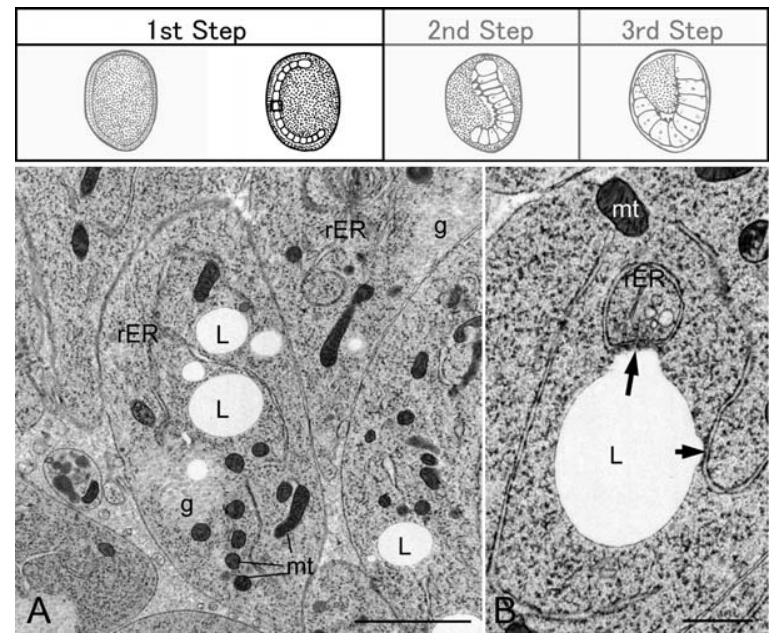

Fig. 3. TEM images of embryonic tissues at the end of the first step of lipid incorporation, the late appendage formation stage (stage 17). (A) Embryonic tissues facing the extraembryonic yolk. (B) High-magnification image of an embryonic cell. The non-shaded panel in the diagram at the top indicates the step of lipid incorporation into the embryo, and the black bold square on the drawing of the embryo indicates the area observed by TEM. Arrows indicate the attachment of lipid droplets to rER. L, lipid droplet; g, glycogen granule; rER, rough ER; mt, mitochondria. Scale bars, $2 \mu \mathrm{m}(\mathrm{A}), 0.6 \mu \mathrm{m}(\mathrm{B})$.

step (Fig. 3), in the late appendage formation stage (stage 17), thick mitochondria and elongated rERs were observed in the cytoplasm. Lipid droplets were also seen, and some of these were attached to the rER (Fig. 3B, arrows).

Fig. 4 shows a sagittal section of the developing caterpillar at the end of the second step, the complete embryonic reversal stage (stage 22). In this stage, embryonic dorsal 


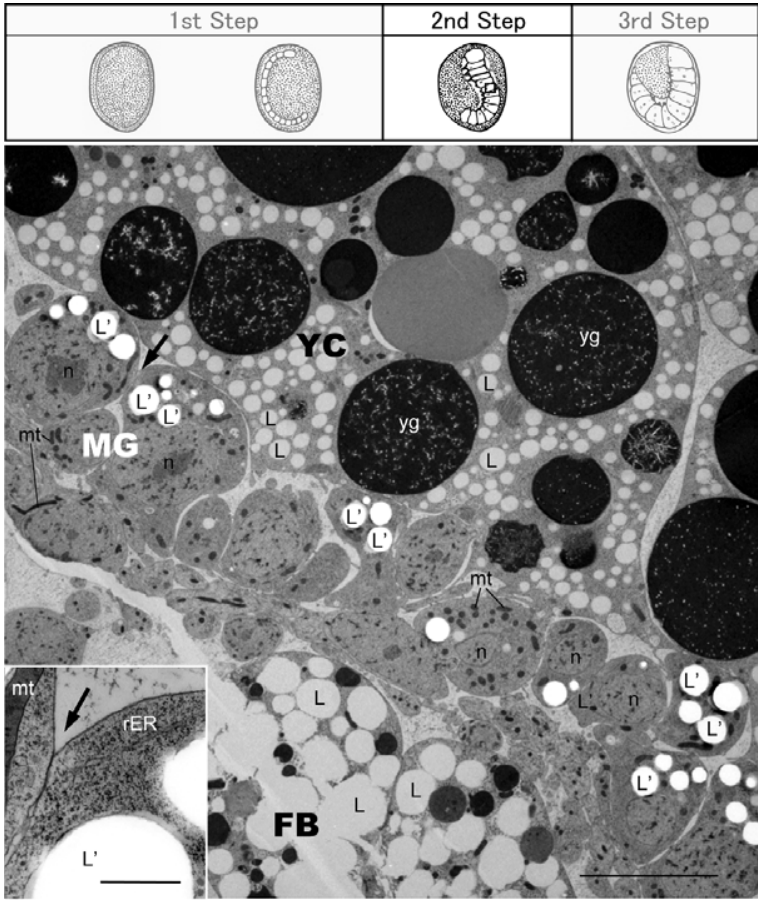

Fig. 4. TEM image of embryonic tissues in the second step of lipid incorporation, the complete embryonic reversal stage (stage 22). The arrow indicates the junction of the intercellular midgut. The inset shows a high-magnification image of the area indicated by the arrow. The non-shaded panel in the diagram at the top indicates the step of lipid incorporation into the embryo, and the black bold square on the drawing of the embryo indicates the area observed by TEM. YC, yolk cell; MG, midgut; FB, fat body; L, lipid droplet; L', large oval structure (a large hole in the ultrathin section); g, glycogen granule; $y g$, yolk granule; $n$, nucleus; $m t$, mitochondria; $r E R$, rough ER. Scale bar, $8 \mu \mathrm{m}$ (in inset, $0.8 \mu \mathrm{m}$ ).

closure had been completed and about half the yolk cells were enclosed inside the midgut lumen without having been digested. Midgut cells were attached to each other and formed the lumen (Fig. 4, inset), but they were still morphologically undifferentiated into cell types such as goblet cells or columnar cells. Several fat bodies were observed in the coelom of the developing caterpillar, and lipid droplets were seen in the yolk cells, midgut, and fat bodies (Fig. 4). Large oval structures in the cells of the midgut were surrounded by substances of the same electron density as the lipid droplets (Fig. 5). When the focus was changed, fringes were observed at the borders of these large oval structures (data not shown), which therefore represented large holes in the ultra-thin sections. Golgi complexes and well-developed rER were observed in the midgut cells (Fig. 5). The rER lumen became wide (Fig. 5C-E, white arrowheads), and vesicular structures were seen in the midgut cells (Fig. 5B-E, black arrowheads). Some of these vesicular structures were derived from the partially expanded $\mathrm{rER}$ (Fig. 5B, white arrow). Moreover, it was observed that the rER-derived vesicular structures were fused with lipid droplets (Fig. 5E, black arrow).

\section{DISCUSSION}

It is generally difficult to prepare insect eggs for electron

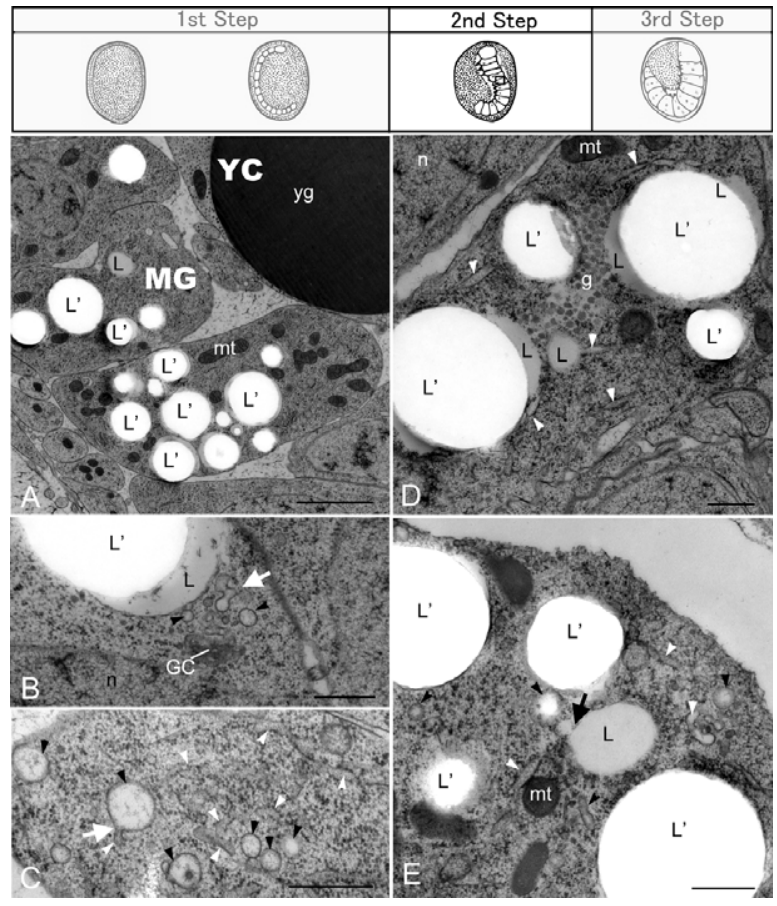

Fig. 5. TEM image of the embryonic tissues in the second step of lipid incorporation, the complete embryonic reversal stage (stage 22). The non-shaded panel in the diagram at the top indicates the step of lipid incorporation into the embryo, and the black bold square on the drawing of the embryo indicates the area observed by TEM. (A) Low-magnification image of a sagittal section of the midgut. Many large oval structures (L') surrounded by a lipid substance are evident in the midgut cells. (B-E) High-magnification images of midgut cells. White arrowheads indicate rER; black arrowheads indicate rERderived vesicular structures; white arrows indicate the connection between $\mathrm{rER}$ and $\mathrm{rER}$-derived vesicular structures; the black arrow indicates the fusion of $\mathrm{rER}$-derived vesicular structures and lipid droplets. YC, yolk cell; MG, midgut; L, lipid droplet; L', large oval structure (large hole in the ultrathin section); n, nucleus; mt, mitochondria; GC, Golgi complex. Scale bars, $2 \mu \mathrm{m}(\mathrm{A}), 0.5 \mu \mathrm{m}(\mathrm{B}-\mathrm{E})$.

microscopy, because they have many nutrients in the cytoplasm, such as lipid droplets and glycogen granules. It is known that chemical fixatives are relatively poor at preserving or preventing the extraction of some classes of molecules, such as sugars and lipids, during the processing steps, and thus procedures may change the dimensions or arrangement of certain cellular components, such as the dimensions of the extracellular space (Chan et al., 1992, 1993, Zechmann et al., 2007). The present study showed that lipid droplets and the surrounding ultrastructure of the cytoplasm are well preserved in eggs of $B$. mori prepared by using high-pressure freezing combined with freeze substitution. High-pressure freezing can fix and preserve the cell structure in its natural state without chemical fixation, making it the most preferable procedure for observing the localization of lipid droplets in the B. mori egg.

Lipid droplets have uniform electron densities in yolk cells and embryonic cells in the first step, and in the yolk cells, midgut, and fat bodies of the developing caterpillar in the second step. It is noteworthy that in the midgut cells in the second step, many large oval structures surrounded by 
lipid substances were observed, in addition to small lipid droplets. We confirmed that the large oval structures were holes in the ultrathin sections. These large holes surrounded with lipid substances were also observed in the midgut in the third step (data not shown). Canavoso and Wells (2000) reported that in fifth-instar larvae of Manduca sexta, the triglyceride pool in the midgut is metabolically active and serves as a reservoir to export diglycerides into the hemolymph. It seems that the lipid substances in the midgut are more unstable than those stored in fat bodies and yolk cells. Therefore, the large oval structures surrounded by lipid substances are presumably lipid droplets. Only the peripheral region of lipid droplets was maintained by the high-pressure freezing and freeze substitution used for TEM specimen preparation; the content in the center of lipid droplet was not retained. According to Miya (1976), there are large vacuoles in midgut tissues at stage 23 prepared by conventional TEM methods. Considering their morphology, it seems that the large vacuoles mentioned by Miya were corresponded to the large oval structures that might have been lipid droplets.

During embryonic development, lipids are involved at all stages from the extraembryonic yolk to the embryo prior to the major energy consumption by the tracheae late in embryogenesis (Yamahama et al., 2008). At the beginning of the first step, there are already some lipid droplets and glycogen granules in the embryonic cells. It seems that these lipid droplets are derived from the oocyte peripheral cytoplasm by superficial cleavage during blastoderm formation. Following the next stage, germband formation (stage 4), however, it seems that the lipid droplets are difficult to incorporate into the embryonic cells in the same manner as at stage 3 , because all the extraembryonic lipid droplets are compartmented in the yolk cells. In order to incorporate lipid into the embryonic cells after stage 4, biogenesis of lipid droplets must occur.

At the end of the first step (stage 17), elongated rER was attached to lipid droplets in embryonic cells. In the second step, well-developed rER and ER-derived vesicular structures were attached to or fused with lipid droplets in midgut cells, and these ER structures thus seem to contribute to the biogenesis of these lipid droplets. It is well known that the ER is closely involved in the biogenesis of lipid droplets in the cytoplasm (Kukulies and Komnick, 1984, van Antwerpen et al., 2005, Rovenek et al., 2006, Kadereit et al., 2008, Ohsaki et al., 2008). Therefore, it seems that the attachment of the elongated $\mathrm{rER}$ to lipid droplets at the end of the first step contributes to the formation of lipid droplets.

Our results suggest that incorporation of lipid droplets in the first step can be subdivided into two stages: the first superficial cleavage of blastoderm formation, and subsequent incorporation from the embryonic cell surface. Observations of lipid incorporation in cells during embryonic development in $B$. mori will help elucidate the correlation between the acquisition of cellular function and morphological cell differentiation during development.

\section{ACKNOWLEDGMENTS}

We are grateful to the Department of Biological Science, Faculty of Science, Nara Women's University for allowing us to use their high-pressure freezer Leica EM-PACT. This work was supported by a Grant-in Aid from the Ministry of Education, Science, and Culture of Japan (18580051).

\section{REFERENCES}

Beenakkers AMT, van der Horst DJ, van Marrewijk WJA (1981) Role of lipids in energy metabolism. In "Energy Metabolism in Insects" Ed by RGH Downer, Plenum Press, New York, pp 53-100

Canavoso LL, Wells MA (2000) Metabolic pathways for diacylglycerol biosynthesis and release in the midgut of larval Manduca sexta. Insect Biochem Mol Biol 30: 1173-1180

Chan FL, Inoue S, Leblond CP (1992) Localization of heparin sulfate proteoglycan in basement membrane by side chain staining with cuprolinic blue as compared with core protein labeling with immunogold. J Histochem Cytochem 40: 1559-1572

Chan FL, Inoue S, Leblond CP (1993) The basement membranes of cryofixed or aldehyde-fixed, freeze-substituted tissues are composed of a lamina densa and do not contain a lamina lucida. Cell Tissue Res 273: 41-52

Kadereit B, Kumar P, Wang WJ, Miranda D, Snapp EL, Severina N, Torregroza I, Evans T, Silver DL (2008) Evolutionarily conserved gene family important for fat storage. Proc Natl Acad Sci USA 105: 94-99

Kukulies J, Komnick H (1984) Lipid transport through the enterocytes of larval Aeshna cyanea (Insecta, Odonata). Eur J Cell Biol 34: 118-129

Miya K (1976) Ultrastructural changes of embryonic cells during organogenesis in the silkworm, Bombyx mori, II. The alimentary canal and the malpighian tubules. J Fac Agr Iwate Univ 13: 95-122

Miya K (2003) The Early Embryonic Development of Bombyx mori. Gendaitosho, Japan

Miya K, Kurihara M, Tanimura I (1972) Changes of fine structures of the serosa cell and the yolk cell during diapause and postdiapause development in the silkworm, Bombyx mori L. J Fac Agr Iwate Univ 11: 51-87

Ohsaki Y, Cheng J, Suzuki M, Shinohara Y, Fujita A, Fujimoto T (2008) Biogenesis of cytoplasmic lipid droplets: From the lipid ester globule in the membrane to visible structure. Biochem Biophys Acta: in press

Ohtsuki Y (1979) Silkworm eggs. In "A General Textbook of Sericulture" Ed by Japanese Society of Sericultural Science, Nihon Sansi Shinbun-Sha, Tokyo, pp 156-173 (in Japanese)

Robenek H, Hofnagel O, Buers I, Rovenek MJ, Troyer D, Severs NJ (2006) Adipophilin-enriched domains in the ER membrane are sited of lipid droplet biogenesis. J Cell Sci 119: 4215-4224

Singh T, Rao V, Jingade AH, Samson MV (2002) Structure and development of silkworm (Bombyx mori) eggs. Sericologia 42: 323-334

van Antwerpen R, Pham DQD, Ziegler R (2005) Accumulation of lipids in insect oocytes. In "Reproductive Biology of Invertebrates, Volume XII, Part B, Progress in Vitellogenesis" Ed by KG Adiyodi, RG Adiyodi RG (Series), AS Raikhel (Volume), Science Publishers, USA, pp 265-287

Yamahama $Y$ (2008) Transmission electron microscopy for insect egg: improvement of specimen preparation methods for the mature egg of silkworm, Bombyx mori. Comp Biochem Physiol 25, 165-170 (in Japanese)

Yamahama Y, Seno K, Hariyama T (2008) Changes in lipid droplet localization during the embryogenesis of the silkworm, Bombyx mori. Zool Sci 25: 580-586

Yamashita O, Yaginuma T (1991) Silkworm eggs at low temperatures: implications for sericulture. In "Insects at Low Temperature" Ed by RE Lee, DL Denlinger, Chapman and Hall, New York, pp 424-445

Zechmann B, Muller M, Zellnig G (2007) Membrane associated qualitative differences in cell ultrastructure of chemically and high pressure cryofixed plant cells. J Struct Biol 158: 370-377

(Received December 24, 2008 / Accepted February 17, 2009) 